\title{
Estilos intelectuais e estratégias de aprendizagem: percepção de professores do ensino fundamental
}

\author{
Francislaine Flâmia Inácio \\ Universidade Estadual de Londrina - Londrina - PR - Brasil \\ Katya Luciane de Oliveira \\ Universidade Estadual de Londrina - Londrina - PR - Brasil \\ Maria Luzia Silva Mariano \\ Universidade Estadual de Londrina - Londrina - PR - Brasil
}

\begin{abstract}
Resumo
Os estilos intelectuais e as estratégias de aprendizagem podem ser eficazes na melhora do desempenho de alunos que apresentam dificuldades para aprender. O objetivo da pesquisa foi averiguar a percepção dos professores acerca dos estilos intelectuais e das estratégias de aprendizagem em alunos do Ensino Fundamental com diagnóstico de Dislexia e TDAH e sem dificuldade escolar. Participaram 23 professores de escolas públicas e foi utilizado um questionário para a coleta de dados. Os resultados indicaram que os professores relataram benefícios em conhecer os estilos e identificaram o uso de estratégias por parte dos alunos. A maioria dos professores não conhecia termos como estratégias de aprendizagem e estilos intelectuais, por isso as respostas foram dadas de acordo com a sua prática de ensino. O estudo buscou entender e discutir alguns aspectos que envolvem a aprendizagem e levar contribuições para o sistema educacional na condição de melhorias para a qualidade de ensino.
\end{abstract}

Palavras-chave: Psicologia cognitiva; Dislexia; Transtorno de Déficit de Atenção e Hiperatividade.

\section{Intellectual styles and learning strategies: perception of fundamental teaching teachers}

\begin{abstract}
Intellectual styles and learning strategies can be effective in improving the performance of students who are struggling to learn. The objective of the research was to investigate teachers' perceptions about intellectual styles and learning strategies in elementary school students with a diagnosis of dyslexia and ADHD and without school difficulties. There were 23 teachers from public schools who participated and a questionnaire was used to collect data. The results indicated that the teachers reported benefits in knowing the styles and identified the use of strategies by the students. Most teachers did not know terms such as learning strategies and intellectual styles, so the answers were given according to their teaching practice. The study sought to understand and discuss some aspects that involve learning and to bring contributions to the educational system in the condition of improvements to the quality of teaching.
\end{abstract}

Keywords: Cognitive psychology; Dyslexia; Attention Deficit Hyperactivity Disorder.

\section{Estilos intelectuales y estrategias de aprendizaje: percepción de profesores de la enseñanza primaria}

\section{Resumen}

Los estilos intelectuales y las estrategias de aprendizaje pueden ser eficaces en la mejora del desempeño de alumnos que presentan dificultades para aprender. El objetivo de la investigación fue averiguar la percepción de los profesores acerca de los estilos intelectuales y de las estrategias de aprendizaje en alumnos de la Enseñanza Primeria con diagnóstico de Dislexia y TDAH y sin dificultad escolar. Participaron 23 profesores de escuelas públicas y se utilizó un cuestionario para la recolecta de datos. Los resultados indicaron que los profesores relataron beneficios en conocer los estilos e identificaron el uso de estrategias por parte de los alumnos. La mayor parte de los profesores no conocían términos como estrategias de aprendizaje y estilos intelectuales, por eso las respuestas contestaron a las preguntas de acuerdo con su práctica de enseñanza. En el estudio se buscó entender y discutir algunos aspectos que abarcan el aprendizaje y llevar contribuciones para el sistema educacional en la condición de mejoras para la calidad de enseñanza.

Palabras clave: Psicología cognitiva; Dislexia; Trastorno por Déficit de Atención con Hiperactividad. 


\section{Introdução}

Os estilos intelectuais e as estratégias de aprendizagem são considerados pela literatura (Amaral, 2007; Dembo, 1994; Emír, 2013; Boruchovitch, 2014; Busnello, Jou, \& Sperb, 2012; Santos \& Boruchovitch, 2011; Zhang \& Sternberg, 2002, 2006; Zhang, 2013, 2015) variáveis importantes na atuação dos professores e, portanto, no processo de aprendizagem do aluno. Conhecer tais temas pode ser necessário no entendimento e intervenção de alunos que apresentam dificuldades para aprender, como é o caso daqueles com Dislexia e Transtorno de Déficit de Atenção/ Hiperatividade-TDAH.

De acordo com Biggs, Kember e Leung (2001) e Felder e Spurlin (2005), os estilos estão relacionados à preferência do indivíduo de processar a informação. A Teoria do Autogoverno Mental apresentado por Zhang e Sternberg (2005), pela primeira vez adotou o termo "estilos intelectuais" para abranger todos os estilos existentes. Tal teoria utilizou-se dos tipos de governo existentes no mundo como metáfora para explicar como as pessoas realizavam o autogoverno mental. Para isso, apresentou trezes estilos que podem ser organizados em três tipos: Tipo I, II e III. O Tipo I envolve os estilos legislativo, judicial, global, hierárquico e liberal. O Tipo II compreende os estilos executivo, conservador, local e monárquico. O Tipo III inclui os estilos oligárquico, anárquico, interno e externo (Fan \& Zhang, 2014; Zhang \& Sternberg, 2005).

Os estilos devem envolver não apenas a aprendizagem dos alunos, mas também os professores, o ensino, a escola/universidade e a administração dos gestores na promoção de estratégias que incentivem o Tipo I de estilos, pois é o que tem se mostrado mais favorável à aprendizagem (Zhang \& Sternberg, 2006). Para que ocorra maior investimento nos estilos do Tipo I, os professores precisam ser mais conscientes do conceito de estilos intelectuais em sua prática docente, assim como os demais gestores, para implementar as politicas institucionais (Zhang \& Sternberg, 2002).

Zhang (2013) afirma que os programas de treinamentos foram quase que exclusivamente projetados para desenvolver o Tipo I de estilos e verificam sucesso na formação desse tipo de estilos intelectuais. Considerando que é necessário aos professores o uso de estratégias específicas para a promoção dos estilos intelectuais do Tipo I que favorecem a criatividade, cabe a eles também incentivar os alunos a desenvolverem estilos adaptativos que seriam benéficos a longo prazo e importantes nos diferentes momentos e contextos (Zhang, 2015). Segundo Emír (2013), os professores devem permitir aos alunos o uso dos estilos intelectuais com o intuito de promover o pensamento crítico e proporcionar o desenvolvimento de novos estilos.

No processo de aprendizagem, além de incentivarem o uso de estilos intelectuais do Tipo I, os professores também podem promover o emprego de estratégias de aprendizagem que facilitem a aquisição, armazenamento e/ou utilização da informação (Amaral, 2007). As estratégias de aprendizagem, de acordo com a Teoria do Processamento da Informação, são ações mentais e comportamentais que auxiliam no processo de aprendizagem por meio de algumas estratégias cognitivas e metacognitivas que favorecem a autorregulação do comportamento (Boruchovitch, 1999).

Segundo Santos e Boruchovitch (2011), para que os professores possam incentivar os alunos no uso das estratégias de aprendizagem, é necessário que eles também saibam como aprender a aprender, de forma eficiente. As pesquisas (Boruchovitch, 2014; Santos \& Boruchovitch, 2011) mostraram despreparo dos professores para intervir junto aos alunos com estratégias de aprendizagem autorregulada, provavelmente devido à deficiência em sua formação. Investir em estratégias para a melhoria do processamento da informação e promover o desenvolvimento metacognitivo é necessário para a formação de professores autorregulados e, por sua vez, alunos conscientes dos seus próprios processos cognitivos. Busnello e cols. (2012) verificaram que após um curso de capacitação em estratégias para professores houve mudanças na metodologia pedagógica e também no uso de estratégias pelos alunos.

De acordo com Dembo (1994), os professores podem contribuir com a melhora das dificuldades de seus alunos. Alguns estudos (Boruchovitch \& Santos, 2015; Perassinoto, Boruchovitch, \& Bzuneck, 2013) indicam que o baixo rendimento escolar de alunos e as dificuldades de aprendizagem podem estar relacionados ao não domínio das estratégias de aprendizagem (cognitivas e metacognitivas).

Segundo Boruchovitch (1999), as estratégias de aprendizagem podem beneficiar alunos com dificuldade escolar. Dessa forma, tais estratégias podem ser ensinadas aos alunos com o objetivo de evitar o fracasso escolar, pois possibilita ao aluno diminuir suas dificuldades e aprender a controlar os fatores que interferem no seu desempenho acadêmico, melhorando sua aprendizagem (Boruchovitch, 1994; Silva \& Sá, 1997). Há indicadores na literatura científica (Capellini \& Oliveira, 2013; Egeland, Johansen, \& Ueland, 2010; Kirby, Silvestri, Allingham, Parrila, \& La Fave, 2008; Knouse, Anastopoulos, \& Dunlosky, 2012) que mostram que alunos com diagnóstico de Dislexia e Transtorno de Déficit de Atenção/ Hiperatividade-TDAH podem apresentar dificuldades no uso de estratégias de aprendizagem; portanto, é importante que o professor conheça sobre os transtornos para poder intervir da melhor forma no desenvolvimento das estratégias.

A Dislexia é um Transtorno Específico de Aprendizagem com prejuízo na leitura que se caracteriza por déficits no reconhecimento de palavras, na decodificação e na compreensão em leitura. O TDAH apesar de não ser um Transtorno Específico de Aprendizagem, acaba por comprometer o desempenho acadêmico por apresentar comportamentos de desatenção e/ou hiperatividade/ impulsividade (American Psychiatric Association, 2014). Nessa perspectiva, Cabral (2013) fez um levantamento sobre estudos relacionados à Dislexia e TDAH no Brasil e verificou que a maior parte das pesquisas se concentra na área de ciências médicas e que na área da educação há uma escassez de material publicado. Dessa forma, o autor destaca a necessidade de políticas públicas voltadas aos alunos com esses transtornos, principalmente com vistas à formação de professores. 
O professor pode ser considerado como figura fundamental no desenvolvimento de estratégias que possibilitem a aprendizagem nos alunos com Dislexia e TDAH. Pimenta (2012) e A. Silva (2015) buscaram conhecer a percepção desses profissionais a respeito dos alunos com Dislexia. As pesquisas mostraram que a maioria dos professores já tinha tido contato com alunos com Dislexia e que apesar de conhecerem o conceito do transtorno, não possuíam informações suficientes sobre o lidar com esses indivíduos. Além disso, verificou-se carência de formação extracurricular sobre o tema. É importante que o professor trabalhe de modo que consiga identificar os sinais e amenizar as dificuldades trazidas pelo transtorno de aprendizagem.

A pesquisa de Silva e Dias (2014) aponta para alguns aspectos importantes ao professor para o trabalho com alunos com TDAH. A motivação e estimulação do professor para com o aluno durante as atividades, além da necessidade de interação entre professor/ professor, aluno/ aluno e professor/ aluno para que ocorra maior motivação e confiança no aprender. Outros aspectos levantados dizem respeito à privacidade do aluno e ao seu ritmo de aprendizagem, o uso de uma metodologia adequada às necessidades do aluno e o aprimoramento constante do professor para que possa fortalecer a sua prática em sala de aula e o desenvolvimento pedagógico. Além disso, o trabalho conjunto entre professor de Sala de Ensino Regular e aqueles de Sala de Recursos Multifuncional são importantes na criação de estratégias que melhor facilitem o aprendizado do aluno (K. Silva, 2015; Silva \& Dias, 2014).

Alguns autores (Amaral, Santos, Rosa, David, \& Przybyszeweski, 2013; Freitas Figueiredo, Bomfim, \& Mendonça, 2010; Santos, Fonseca, Turbino, Castro, \& Brandi, 2013; Seno, 2010) realizaram entrevistas com professores a respeito de temas relacionados ao TDAH e verificaram que parte destes não tem conhecimento sobre o transtorno, mas que a atuação desse profissional era fundamentada em sua prática em sala de aula. Com base no que foi exposto, este trabalho buscou investigar a percepção dos professores acerca dos estilos intelectuais e das estratégias de aprendizagem em alunos matriculados no Ensino Fundamental com diagnóstico de Dislexia, TDAH e sem dificuldade.

\section{Método}

\section{Participantes}

A amostra foi composta de 23 professores do Ensino Fundamental $\left(2^{\circ}\right.$ ao $9^{\circ}$ ano), sendo que $30,4 \%(n=7)$ atuavam em Sala de Ensino Regular, 60,9\% ( $n=14)$ em Sala de Recursos Multifuncional e 8,7\% (n=2) em ambas as salas. A média de tempo de trabalho dos professores foi de 15 anos e um mês $(D P=10,87)$, sendo o tempo mínimo de um ano e o máximo de 35 anos. A média de idade dos professores foi de 40 anos e 3 meses $(D P=8,28)$, com idade mínima de 24 anos e máxima de 59 anos.

\section{Instrumentos}

O instrumento aplicado para a coleta de dados foi um questionário, elaborado pelas autoras, com 10 questões dissertativas que incluíam os seguintes assuntos: definição sobre dificuldade escolar, Transtorno de Aprendizagem, Dislexia e TDAH, diferenças entre alunos com Dislexia e TDAH em memória, contribuição dos estilos intelectuais para o aprendizado dos alunos, identificação das estratégias de aprendizagem utilizadas pelos alunos, diferenças entre alunos com Dislexia, TDAH e sem dificuldade escolar em estilos intelectuais e estratégias de aprendizagem e como promover nos alunos o desenvolvimento dos estilos intelectuais e das estratégias de aprendizagem necessários para o processo ensino-aprendizagem.

\section{Procedimentos}

A coleta de dados foi iniciada após a aprovação pelo Comitê de Ética em Pesquisa - CEP, da Universidade Estadual de Londrina - UEL, sob o protocolo $n^{\circ} 854.426 / 2014$ e se respaldou na Resolução 466/12 do Conselho Nacional de Saúde e suas complementares (Resolução n ${ }^{\circ} 466$, 2012). Os questionários foram entregues a professores de 55 escolas públicas, municipais e estaduais, de três cidades do Norte do Paraná. Em alguns momentos o pesquisador fez o contato direto com o professor e, em outros, a equipe pedagógica da escola ficou encarregada de conversar com o profissional sobre a pesquisa. Para a aplicação do questionário foram entregues os Termos de Consentimento Livre e Esclarecido - TCLEs juntamente com o questionário e combinado um período para a devolução de ambos. Vale ressaltar que a pesquisa também foi realizada com professores que atuavam em Salas de Recursos Multifuncional-Tipo I devido e estas salas atenderem alunos com diagnóstico de Dislexia e TDAH. Para a análise dos dados utilizou-se o método survey, no qual foi feito o agrupamento e categorização das respostas, seguido do levantamento de frequência e porcentagem.

\section{Resultados}

As questões foram analisadas e as categorias criadas e agrupadas de acordo com a similaridade nas respostas dos participantes. Com o intuito de atender o objetivo proposto, cinco questões respondidas pelos professores foram investigadas neste estudo. Os resultados obtidos serão descritos a seguir. A primeira questão mostra a concepção dos professores sobre Dislexia e Transtorno de Déficit de Atenção/ Hiperatividade - TDAH. Nessa pergunta houve um equilíbrio entre praticamente todas as porcentagens, sete professores $(30,4 \%)$, relataram que "a Dislexia é um Transtorno Específico de Aprendizagem de origem neurobiológica, com dificuldades no reconhecimento de palavras e decodificação de letras e o TDAH é um transtorno neurobiológico 
do desenvolvimento e apresenta sintomas de desatenção e hiperatividade". Outros seis professores $(26,1 \%)$ responderam que a "Dislexia está relacionada com dificuldade de leitura, escrita e compreensão e o TDAH com dificuldade de concentração, impulsividade, hiperatividade", sendo que o mesmo número de professores $(n=6 ; 26,1 \%)$ diss que a "Dislexia é um Transtorno Específico de Leitura e o TDAH é um Transtorno de Déficit de Atenção-Hiperatividade, transtorno neurobiológico ou transtorno de comportamento que se caracteriza por sintomas de desatenção, hiperatividade e impulsividade". Apenas três professores (13,0\%) relataram que a Dislexia e o TDAH eram "transtornos neurobiológicos" e um professor $(4,3 \%)$ caracterizou os transtornos como "fatores que atrapalham o aprendizado do aluno".

A segunda pergunta buscou saber de que forma o conhecimento do professor sobre os estilos intelectuais poderia contribuir para o aprendizado do aluno. As respostas estão dispostas na Tabela 1.

Tabela 1. Categorização a respeito da percepção do professor sobre os estilos intelectuais para o aprendizado do aluno.

\begin{tabular}{|c|c|c|}
\hline Respostas & $F$ & $\%$ \\
\hline Entender como intervir no aluno e facilitar a aprendizagem & 6 & 26,1 \\
\hline Criar/ modificar/ usar estratégia que melhor se adeque ao aluno & 6 & 26,1 \\
\hline Conhecer cada aluno em suas especificidades e trabalhar suas dificuldades & 4 & 17,4 \\
\hline Saber o interesse do aluno & 3 & 13,0 \\
\hline Trabalhar de acordo com o estilo do aluno para promover a aprendizagem & 2 & 8,7 \\
\hline Verificar qual estilo é mais favorável à aprendizagem & 1 & 4,3 \\
\hline Não respondeu & 1 & 4,3 \\
\hline Total & 23 & 100,0 \\
\hline
\end{tabular}

Nota. F: frequência.

Tabela 2. Categorização acerca de como o professor poderia promover nos alunos o desenvolvimento de estilos intelectuais necessários à eficácia no processo de ensino/aprendizagem.

\begin{tabular}{rcc}
\hline & $F$ & $\%$ \\
\hline Respostas & 8 & 34,8 \\
\hline Atendificando como o aluno aprende e utilizando recurso didático metodológico & 6 & 26,1 \\
\hline Explorando as vias sensoriais & 3 & 13,0 \\
\hline Incentivando e motivando os alunos para aprenderem & 1 & 4,3 \\
\hline Apresentando os estilos intelectuais aos alunos & 1 & 4,3 \\
\hline Estimulando a habilidade de leitura e a expressão verbal & 1 & 4,3 \\
\hline Regras, estabelecimento de rotina, aumento da autoestima e autoconceito & 1 & 4,3 \\
\hline Auxílio da família e de atividades acadêmicas e sociais & 1 & 4,3 \\
\hline Intervindo em áreas afetivo/emocional, motora e cognitiva & 1 & 4,3 \\
\hline
\end{tabular}

Nota. F: frequência.

Nesta análise foi possível perceber um empate entre duas categorias de resposta. A maioria dos professores informou que os estilos intelectuais poderiam contribuir para entender como intervir no comportamento do aluno e facilitar a sua aprendizagem ( $n=6 ; 26,1 \%)$, assim como na criação, modificação e uso de estratégias que melhor se adequassem ao aluno ( $n=6 ; 26,1 \%)$. Apenas um $(4,3 \%)$ professor respondeu que conhecer sobre os estilos proporcionaria verificar qual é o mais favorável à aprendizagem, e outro $(4,3 \%)$ não respondeu.

A terceira questão procurou saber como o professor poderia promover nos alunos o desenvolvimento de estilos intelectuais necessários à eficácia no processo de ensino/ aprendizagem. As respostas dos professores estão categorizadas na Tabela 2. 
Nesta pergunta, a maior parte respondeu $(n=8$; $34,8 \%$ ) que promoveria o desenvolvimento de estilos intelectuais identificando a forma como o aluno aprende e fazendo uso de uma diversidade de recursos didático- metodológicos. Outros professores afirmaram que a melhor forma de desenvolver os estilos intelectuais necessários à aprendizagem seria apresentando tais estilos aos alunos $(4,3 \%)$ ou por meio do incentivo e motivação para aprender $(4,3 \%)$, da estimulação da habilidade de leitura e expressão verbal $(4,3 \%)$, do estabelecimento de regras, rotina e aumento da autoestima e autoconceito (4,3\%), do auxílio da família e de atividades acadêmicas e sociais (4,3\%) e da intervenção em áreas afetivo/emocional, motora e cognitiva $(4,3 \%)$. Estas respostas foram as com índices mais baixos de porcentagem.
A quarta questão buscou saber quais estratégias de aprendizagem o professor percebia em seu aluno. As respostas se encontram dispostas na Tabela 3.

Os dados apresentados na Tabela 3 indicaram que $30,4 \%(n=7)$ dos professores relataram que seus alunos fazem uso de estratégias de sublinhar, anotar, realizar pesquisas na internet, reler e pedir ajuda a um mediador. Outro professor afirmou que os alunos usam como estratégia de aprendizagem participar das aulas $(n=1 ; 4,3 \%)$.

A última pergunta buscou entender como o professor poderia promover nos alunos o desenvolvimento de estratégias de aprendizagem importantes para um melhor desempenho acadêmico. A Tabela 4 mostra as respostas dos professores em categorias.

Tabela 3. Categorização a respeito de quais estratégias de aprendizagem o professor percebia em seu aluno.

\begin{tabular}{|c|c|c|c|}
\hline & Respostas & $F$ & $\%$ \\
\hline \multicolumn{2}{|r|}{ Sublinhar/ anotar/ realizar pesquisas na internet/ reler/ pedir ajuda a um mediador } & 7 & 30,4 \\
\hline & Estratégias de ensino & 6 & 26,1 \\
\hline & Não apresentam estratégias & 4 & 17,4 \\
\hline & Usar técnicas de memorização & 3 & 13,0 \\
\hline & Desenhar/ imagens & 2 & 8,7 \\
\hline & Participar das aulas & 1 & 4,3 \\
\hline Total & & 23 & 100,0 \\
\hline
\end{tabular}

Nota. F: frequência.

Tabela 4. Categorização acerca de como o professor poderia promover nos alunos o desenvolvimento de estratégias de aprendizagem importantes para um melhor desempenho acadêmico.

\begin{tabular}{ccc}
\hline Respostas & $F$ & $\%$ \\
\hline $\begin{array}{c}\text { Realizando atendimento individualizado, identificando as particularidades de cada aluno e } \\
\text { atividades de interesse e utilizando metodologia de ensino diversificada }\end{array}$ & 8 & 34,8 \\
\hline Propondo atividades de incentivo à leitura e expressão verbal & 4 & 17,4 \\
\hline Apresentando as estratégias ao aluno para que escolha a de sua preferência & 3 & 13,0 \\
\hline Conhecendo os estilos de aprendizagem do aluno e motivando-o para o aprendizado e uso de & 3 & 13,0 \\
\hline estratégias & 2 & 8,7 \\
\hline Intervindo na capacidade intelectual do aluno e nas funções cognitivas & 1 & 4,3 \\
\hline Levando o aluno a entender a necessidade do uso das estratégias & 1 & 4,3 \\
\hline Promovendo o treinamento de estratégias & 1 & 4,3 \\
\hline Total & 23 & 100,0 \\
\hline
\end{tabular}

Nota. F: frequência. 
Analisando a quinta pergunta que os professores responderam sobre como poderiam promover nos alunos o desenvolvimento de estratégias de aprendizagem importantes para o desempenho acadêmico, verificou-se que a maioria ( $n=8 ; 34,8 \%)$ informou que a melhor forma seria por meio do atendimento individualizado, identificando as particularidades de cada aluno, suas atividades de interesse e utilizando uma metodologia de ensino diversificada. O menor número de professores afirmou que isso seria possível por meio de vivências, experiências, reflexões e atitudes em relação à aprendizagem $(4,3 \%)$ ou levando o aluno a entender a necessidade do uso das estratégias $(4,3 \%)$ ou, ainda, com o treinamento dessas estratégias de aprendizagem (4,3\%). A seguir será abordada a discussão dos resultados obtidos a partir do referencial teórico apresentado neste trabalho.

\section{Discussão}

Quanto à amostra de professores, foram entregues questionários àqueles responsáveis por Sala de Recursos Multifuncional e por Sala de Ensino Regular, sendo que o primeiro grupo se mostrou mais disponível em responder o instrumento. Isso pode ter ocorrido, devido ao interesse destes profissionais em alternativas que contribuam para a melhora no desempenho dos alunos atendidos, tendo em vista a falta de uma equipe multiprofissional para dar suporte ao trabalho avaliativo e interventivo. Em contrapartida, a maior parte dos professores de Sala de Ensino Regular alegou impossibilidade de responder ao questionário, devido ao tempo. Segundo K. Silva (2015) e Silva e Dias (2014) é necessário que os professores de Sala de Recursos Multifuncional e da Sala de Ensino Regular realizem um trabalho conjunto, buscando estratégias que melhor favoreçam o aprendizado do aluno.

Em relação à análise da percepção dos professores quanto aos temas discutidos na presente pesquisa. No que se refere à diferença entre Dislexia e TDAH, verificou-se que a maioria dos professores apresentou os conceitos esperados sobre os transtornos. Em relação ao TDAH, as pesquisas de Amaral e cols. (2013), Freitas e cols. (2010), Santos e cols. (2013) e Seno (2010) constataram que o professor não possuía embasamento teórico sobre o transtorno, o que não foi observado na presente pesquisa. No caso da Dislexia, os trabalhos de Pimenta (2012) e A. Silva (2015) concluíram que os professores conheciam o conceito sobre o transtorno, mas não tinham informações suficientes para lidar com os alunos. Vale ressaltar que a maioria dos professores lecionava em Sala de Recursos Multifuncional e, por isso, eram especializados na área de educação especial e afins. Além disso, outro fato importante é que os professores puderam realizar consulta para responder aos questionários, o que pode ter interferido na precisão das respostas.

No que concerne à questão que analisou de que forma o conhecimento dos professores sobre os estilos intelectuais poderia contribuir para o aprendizado do aluno, os professores trouxeram respostas que de fato mostraram entendimento dos benefícios dos estilos intelectuais. Dentre elas, saber como intervir e facilitar a aprendizagem, auxiliar na criação, modificação e uso de estratégias, conhecer cada aluno em suas especificidades e trabalhar suas dificuldades, além de outras. Aos professores foi apresentado apenas o conceito do construto. De acordo com Zhang e Sternberg (2002), os professores devem ser conscientes do conceito de estilos intelectuais em sua prática docente. Zhang (2013) e Zhang e Sternberg (2006) também afirmaram que a aprendizagem dos estilos deve envolver os alunos, os professores, o ensino, a escola/universidade e os gestores. Além disso, cabe aos professores incentivar o uso dos estilos do Tipo I, eficazes no desenvolvimento da aprendizagem.

No que se refere à maneira como o professor poderia promover nos alunos o desenvolvimento de estilos intelectuais necessários à eficácia no processo de ensino/aprendizagem, as respostas foram baseadas no conhecimento adquirido em sua prática pedagógica diária. Algumas respostas relataram identificar como o aluno aprende, realizar atendimento individualizado e com atividades específicas e diferenciadas. Em vista disso, as pesquisas de Emír (2013) e Zhang (2015) trouxeram algumas contribuições ao professor da forma como incentivar nos alunos a diversidade de estilos e favorecer a aprendizagem. Dessa forma, os autores propuseram que os professores promovessem os estilos do Tipo I, importantes no desenvolvimento da criatividade e do pensamento crítico. Além disso, que estimulassem o desenvolvimento de novos estilos que pudessem vir a ser benéficos a longo prazo.

$\mathrm{Na}$ questão sobre quais estratégias de aprendizagem o professor percebia em seu aluno, a maioria identificou estratégias de aprendizagem como sublinhar, anotar, pesquisar, reler e pedir ajuda. Alguns professores também informaram que seus alunos não apresentavam estratégias de aprendizagem, e outros relataram apenas estratégias de ensino. As pesquisas de Boruchovitch (2014) e Santos e Boruchovitch (2011) mostraram que devido ao desconhecimento do tema, os professores acabam por confundir as estratégias de aprendizagem com as estratégias de ensino. Identificar e incentivar o uso das estratégias de aprendizagem nos alunos depende da capacitação dos professores. Busnello e cols. (2012) afirmaram que o treinamento dos docentes em estratégias de aprendizagem melhora a prática pedagógica e o desenvolvimento acadêmico dos alunos.

Por fim, na questão relacionada à forma como os professores poderiam promover nos alunos o desenvolvimento de estratégias importantes para o desenvolvimento acadêmico, a maioria relatou que seria realizando atendimento individualizado, identificando as particularidades de cada aluno e atividades do seu interesse e utilizando metodologia diversificada. Tais ações não necessariamente indicam o desenvolvimento das estratégias de aprendizagem. As respostas de alguns professores demonstraram como promover as estratégias de aprendizagem nos alunos, dentre elas, apresentar as estratégias ao estudante para que escoIha a de sua preferência, levá-lo a entender a necessidade do uso de estratégias e promover o seu treinamento. De 
acordo com Amaral (2007), o professor pode auxiliar o aluno na identificação de estratégias (cognitivas e metacognitivas) que facilitem o processo de aprendizagem, assim como na capacidade de estabelecer metas, planejar e monitorar o comportamento, de forma que garanta o desenvolvimento pessoal, intelectual e acadêmico deste aluno (Almeida, 2002; Gomes \& Boruchovitch, 2005; Souza, 2010). Apesar de a maioria dos professores ter identificado as estratégias de aprendizagem utilizadas pelos alunos, abordado em questão anterior, isso não significa conhecimento da teoria pois, como foi mencionado, os professores tiveram dificuldades em relatar como promoveriam o desenvolvimento de tais estratégias.

Alguns fatores podem ter contribuído para o desempenho dos professores ao responder aos questionários. Como foi mencionado, a maior parte dos professores lecionava em Sala de Recursos Multifuncional; portanto, possuía formação extracurricular na área de educação especial. Além disso, em 2014, os professores das escolas municipais de uma das cidades em que foi realizada a pesquisa passaram por capacitação sobre as estratégias de aprendizagem, promovida pelo departamento de Psicologia da Universidade Estadual de Londrina, o que pode ter contribuído para um maior entendimento sobre o assunto. Outra questão pode ter sido o fato de os professores terem tido um tempo para responder ao questionário, o que de certa forma pode ter possibilitado a resposta sob consulta.

\section{Considerações Finais}

A pesquisa buscou averiguar a percepção dos professores em relação a esses temas, visto que estes são os principais profissionais da área da educação. Nos conceitos relacionados à Dislexia e TDAH, a maioria dos professores demonstrou conhecimento sobre o assunto. No que se refere aos estilos intelectuais, os professores relataram benefícios que o conhecimento sobre os estilos poderia proporcionar ao aprendizado do aluno e em relação às estratégias de aprendizagem a maioria dos professores identificaram as estratégias utilizadas por seus alunos. Quanto a promover o desenvolvimento de estilos e estratégias nos alunos, as respostas dos professores foram de acordo com a sua prática de ensino.

O objetivo do questionário não foi avaliar o nível de conhecimento do professor, visto que foi informado ao profissional o conceito de termos como estilos intelectuais e estratégias de aprendizagem. A pesquisa buscou entender como o conhecimento dessas variáveis poderia interferir na atuação do professor em sala de aula. Vale ressaltar que durante a revisão bibliográfica foi verificada uma escassez de pesquisas que investigassem os estilos intelectuais e as estratégias de aprendizagem relacionadas ao professor. Levar tais temas para as escolas é de suma importância no processo de aprendizagem, pois podem contribuir no planejamento interventivo, visto que tais assuntos são pouco abordados e, alguns deles, nem mesmo são mencionados.
Apesar de os professores conhecerem o tema Dislexia e TDAH, além da maioria da amostra lecionar em Sala de Recursos Multifuncional, sabe-se da dificuldade para trabalhar com os alunos em sala de aula, seja pela falta de estrutura física ou de políticas públicas educacionais que defendam os direitos desta população. Deste modo, o papel do professor se mostra ainda mais importante no desenvolvimento dos alunos com diagnóstico, sendo que investir em sua formação pode ser visto como fator necessário na superação das dificuldades acadêmicas dos educandos.

A carência de políticas públicas educacionais relacionadas aos alunos com Dislexia e TDAH tem como consequência o hiper diagnóstico e, por conseguinte a medicalização escolar. A falta de uma equipe multidisciplinar que contribua com o processo de diagnóstico faz com que alunos com dificuldade escolar sejam taxados com alterações em funções cognitivas. Tal situação é por vezes aceita pelos pais e pela escola por proporcionar ao aluno um atendimento educacional especializado em Salas de Recursos Multifuncional. Dessa forma, a necessidade de tal atendimento, somada à impossibilidade que isso seja feito em Sala Ensino Regular, dadas as condições atuais da educação, faz com que o diagnóstico seja utilizado como solução ao problema do fracasso escolar. É preciso ir além e compreender as demandas sociais, a medicalização envolve o processo de patologização dos problemas educacionais, por isso, cabe aos profissionais da educação buscar o rompimento com essa patologização, considerando as particularidades da educação e contribuindo para que a escola cumpra o seu papel social (Meira, 2012).

\section{Referências}

Almeida, L.S. (2002). Facilitar a aprendizagem: ajudar os alunos a aprender e a pensar. Psicologia Escolar e Educacional, 6(2), 155165.

Amaral, A.B., Santos, C.O., Rosa, C. K., David, M.C. \& Przybyszeweski, T. (2013). A formação do professor para trabalhar com crianças que apresentam diagnóstico de TDAH no ensino fundamental I na Rede Municipal de Ensino de Curitiba. Ensaios Pedagógicos. Recuperado: 7 mar. 2016. Disponível: http://www.opet.com.br/ faculdade/revista-pedagogia/pdf/n6/ARTIGO-MONICA.pdf

Amaral, V.L. (2007). Estratégias e estilos de aprendizagem: a aprendizagem no adulto. Em: V. L. Amaral. Psicologia da educação (pp. 1-20). Natal: EDUFRN.

American Psychiatric Association [APA] (2014). Manual diagnóstico e estatístico de transtornos mentais: DSM-5 (5 $5^{\mathrm{a}}$ ed.) Porto Alegre: Artmed.

Biggs, J.B., Kember, D., \& Leung, D. (2001). The revised twofactor study process questionnaire: R-SPQ-2F. British Journal of Educational Psychology, 71(1), 133-149. 
Boruchovitch, E. (1994). As variáveis psicológicas e o processo de aprendizagem: uma contribuição para psicologia escolar. Psicologia: Teoria e Pesquisa, 10(1), 129-139.

Boruchovitch, E. (1999). Estratégias de aprendizagem e desempenho escolar: considerações para a prática educacional. Psicologia: Reflexão e Crítica, 12(2), 361-376.

Boruchovitch, E. (2014). Autorregulação da aprendizagem: contribuições da psicologia educacional para a formação de professores. Psicologia Escolar e Educacional, 18(3), 401-409.

Boruchovitch, E. \& Santos, A.A.A. (2015). Psychometric Studies of the Learning Strategies Scale for University Students. Paidéia, 25(60), 19-27.

Busnello, F.B., Jou, G.I., \& Sperb, T.M. (2012). Desenvolvimento de habilidades metacognitivas: capacitação de professores de ensino fundamental. Psicologia: Reflexão e Crítica, 25(2), 311-319.

Cabral, G.M. (2013). A Alfabetização de crianças com patologia de Dislexia e/ou TDAH. Ensaios Pedagógicos. Recuperado: 7 mar. 2016. Disponível: http://www.opet.com.br/faculdade/revistapedagogia/pdf/n5/ARTIGO-GILSON.pdf

Capellini, S.A. \& Oliveira, A.M. (2013). Learning strategies evaluation in students with attention deficit hyperactivity disorder. Education Sciences \& Society, 4(2), 53-64.

Dembo, M.H. (1994). Applying educational psychology (5a ed.). New York: Longman.

Egeland, J., Johansen; S.N., \& Ueland, T. (2010). Do low-effort learning strategies mediate impaired memory in ADHD? Journal of Learning Disabilities, 43(5), 430-440.

Emír, S. (2013). Contributions of teachers' thinking styles to critical thinking dispositions (Istanbul-Fatih Sample). Educational Sciences: Theory \& Practice, 13(1), 337-347.

Fan, J. \& Zhang, L.F. (2014). The role of perceived parenting styles in thinking styles. Learning and Individual Differences, 32, 204-211.

Felder, R.M. \& Spurlin, J.E. (2005). Applications, reliability, and validity of the index of learning styles. International Journal of Engineering Education, 21(1), 103-112.

Freitas, J.S., Figueiredo, K.C., Bomfim N.R., \& Mendonça T.F.R. (2010). TDAH: nível de conhecimento e intervenção em escolas do município de Floresta Azul, Bahia. Gerais: Revista Interinstitucional de Psicologia, 3(2), 175-183.

Gomes, M.A.M.; Boruchovitch, E. (2005). Desempenho no jogo, estratégias de aprendizagem e compreensão na leitura. Psicologia: Teoria e Pesquisa, 21(3), 319-326.
Kirby, J.R., Silvestri R., Allingham, B.H., Parrila, R., \& La Fave, C.B. (2008). Learning strategies and study approaches of postsecondary students with dyslexia. Journal of Learning Disabilities, 41(1), 8596.

Knouse, L.E., Anastopoulos, A.D., \& Dunlosky, J. (2012). Isolating metamemory deficits in the self-regulated learning of adults with ADHD. Journal of Attention Disorders, 16(8), 650-660.

Meira, M.E.M. (2012). Para uma crítica da medicalização na educação. Psicologia Escolar e Educacional, 16(1), 135-142.

Perassinoto, M.G.M., Boruchovitch, E., \& Bzuneck, J.A. (2013). Estratégias de aprendizagem e motivação para aprender de alunos do ensino fundamental. Avaliação Psicológica, 12(3), 351359.

Pimenta, D.C.F.G. (2012). Dislexia: um estudo sobre a percepção de professores do ensino fundamental. Em Centro de Ensino, Pesquisa, Extensão e Atendimento em Educação Especial (Org.), Anais do V Seminário Nacional de Educação Especial, IV Encontro de Pesquisadores em Educação Especial e Inclusão Escolar (pp. 1-15). Uberlândia: CEPEA.

Resolução $n^{\circ}$ 466, de 12 de dezembro de 2012 (2012, 12 de dezembro). Aprova as diretrizes e normas regulamentadoras de pesquisas envolvendo seres humanos. Ministério da Saúde: Diário Oficial da União, n 112, Seção 1, pp.59-62.

Santos, A.F., Fonseca, F.V.S., Turbino, M.L., Castro, P.J.L., \& Brandi, M.T. (2013). O papel da escola e do professor no processo de aprendizagem em crianças com transtorno de déficit de atenção e hiperatividade (TDAH). Revista Univiçosa, 5(1), 421-426.

Santos, O.J.X. \& Boruchovitch, E. (2011). Estratégias de aprendizagem e aprender a aprender: concepções e conhecimento de professores. Psicologia: Ciência e Profissão, 31(2), 284-295.

Seno, M.P. (2010). Transtorno do déficit de atenção e hiperatividade (TDAH): o que os educadores sabem? Revista Psicopedagogia, 27(84), 334-343.

Silva, A.L. (2015). Dislexia: dificuldade de aprendizagem e o papel da escola na compreensão e atendimento do aluno com transtorno na linguagem oral e escrita. Revista Eventos Pedagógicos: Desigualdade e Diversidade Étnico-Racial na Educação Infantil, 6(4), 22-33.

Silva, A.L. \& Sá, I. (1997). Saber estudar e estudar para saber. Portugal: Porto Editora.

Silva, K.V.P. (2015). Transtorno do déficit de atenção e hiperatividade (TDAH): um olhar pedagógico. Revista Eventos Pedagógicos: Desigualdade e Diversidade Étnico-Racial na Educação Infantil, 6(4), 223-231. 
Silva, S.B. \& Dias, M.A.D. (2014). TDAH na escola estratégias de metodologia para o professor trabalhar em sala de aula. Desigualdade e Diversidade Étnico-Racial na Educação Infantil, 5(4), 105-114.

Souza, L.F.N.I. (2010). Estratégias de aprendizagem e fatores motivacionais relacionados. Educar, 36, 95-107.

Zhang, L.F. (2013). The malleability of intellectual styles. New York: University Press.
Zhang, L.F. (2015). Fostering successful intellectual styles for creativity. Asia Pacific Education Review, 16(2), 183-192.

Zhang, L.F. \& Sternberg, R.J. (2002). Thinking styles and teacher characteristics. International Journal of Psychology, 37(1), 3-12.

Zhang, L.F. \& Sternberg, R.J. (2005). A threefold model of intellectual styles. Educational Psychology Review, 17(1), 1-53.

Zhang, L.F. \& Sternberg, R.J. (2006). The nature of intellectual styles. Mahwah, NJ: Erlbaum.
Recebido em: 07 de junho de 2016 Aprovado em: 15 de dezembro de 2016

\section{Sobre os autores}

Francislaine Flâmia Inácio (laine_inacio@hotmail.com)

Psicóloga. Especialização em Psicologia Clínica em Neurologia Infantil e Neuropsicologia aplicada à Neurologia Infantil pela Universidade Estadual de Campinas. Mestranda em Educação pelo Programa de Pós-graduação em Educação da Universidade Estadual de Londrina. Bolsista CAPES.

Katya Luciane de Oliveira (katyauel@gmail.com)

Psicóloga, Mestre em Psicologia pelo Programa de Estudos de Pós-graduação Stricto-Sensu em Psicologia da Universidade São Francisco. Doutora em Psicologia, Desenvolvimento Humano e Educação pela Faculdade de Educação da Unicamp. Professora do Programa de Pósgraduação Stricto Sensu em Educação da Universidade Estadual de Londrina. Professora adjunta do curso de Psicologia da Universidade Estadual de Londrina, Centro de Ciências Biológicas, Departamento de Psicologia e Psicanálise, Laboratório de Avaliação e Pesquisa Psicológica/ LAPPSIC. Bolsista produtividade do CNPq.

Maria Luzia Silva Mariano (malumariano@yahoo.com.br)

Graduada em Ciências Sociais com habilitação em Licenciatura pela Universidade Federal de Alfenas. Especialização em Sociologia e Ensino de Sociologia pelo Centro Universitário Claretiano. Mestre e doutoranda em Educação pelo Programa de Pós-graduação da Universidade Estadual de Londrina.

O artigo foi elaborado a partir da dissertação de Francislaine Flâmia Inácio, intitulada "Memória, Estilos Intelectuais e Estratégias de Aprendizagem: estudando os Transtornos do Neurodesenvolvimento em alunos do Ensino Fundamental e percepção de seus Professores". Universidade Estadual de Londrina, 2016. Apoio: Coordenação de Aperfeiçoamento de Pessoal de Nível Superior (Edital PPEdu/UEL n. 002/2014). 